\title{
A Novel Approach that Ameliorates Motor Disabilities and Improves Range of Motion of Joints; Comparison with Proprioceptive Neuromuscular Facilitation
}

\author{
Vinicius Monteiro Diederichs* \\ Visiting Kinesiologist at John Cabot University, Italy \\ *Corresponding author: info@monteirodiederichs.com
}

\begin{abstract}
A novel approach significantly more effective both in ameliorating motor disabilities and in improving range of motion (ROM) of joints when compared with the current standard approach, proprioceptive neuromuscular facilitation (PNF). A total of 56 participants - of both genders, BMI normal, mean age 35 (range 21-68 years), exhibiting lumbopelvic extensor stiffness (assessed by performance of the deep squat test) - engaged in two experiments. Joint ROM was evaluated before and after treatment in each experiment. Participants were photographed performing the deep squat test and their joint ROM calculated with Kinovea software to obtain the data. In Experiment 1, twenty-six participants were randomly divided into two groups in order to compare the degree of improvement in ROM. One group was treated using the novel approach and the control group with PNF, both for three minutes duration. Results: statistically the effect size of the novel approach proved greater compared to the control PNF. In Experiment 2, thirty participants were treated only with the novel approach, motor disability being assessed with the Oswestry Disability Index (ODI) before and after five to seven days. Results: the novel approach proved significantly effective in ameliorating motor disabilities. Conclusion: the novel treatment is effective both in improving ROM of joints and in ameliorating motor disabilities. Moreover, in Experiment 2 the participants were assessed five to seven days after treatment, demonstrating that the improvement in ROM as well as the amelioration of motor disabilities is long lasting. Additionally, the degree of improvement in joint ROM observed in Experiment 1 finds confirmation in Experiment 2. Strikingly, after performance of the novel approach all the participants reported a pleasant sensation of lightness in walking - a phenomenon termed sensory attenuation, the term signifying the matching between sensory prediction and outcome.
\end{abstract}

Keywords: joint stiffness, capability, affordance, muscle spindles, myotatic reflex, recalibration, joint impedance

Cite This Article: Vinicius Monteiro Diederichs, "A Novel Approach that Ameliorates Motor Disabilities and Improves Range of Motion of Joints; Comparison with Proprioceptive Neuromuscular Facilitation." American Journal of Sports Science and Medicine, vol. 6, no. 1 (2018): 15-21. doi: 10.12691/ajssm-6-1-4.

\section{Introduction}

A novel approach, termed Roman Press (RP), designed to increase joint range of motion (ROM) and also to ameliorate motor disabilities is presented here. The effects are complementary to the paradoxical swaying equilibrium (PSE) approach (in press). Conventionally, clinicians use a squared-static position of the body in the attempt to increase ROM of the lumbar-pelvic-hip complex, see Figure 1. Stretching of muscles (by maintaining a timed passive position of the body segments) is currently used to decrease excessive stiffness and to improve ROM of joints [1]. This contrasts however with previous studies which have established that, when a muscle is held extended for several seconds, the muscle spindles lose their sensitivity as a result of slackness on the spindles themselves, a phenomenon termed thixotropy [2]. Thixotropy is detrimental to the body's proprioception and to the myotatic reflex [3]. It follows that recalibration of muscle spindles is possible only by performing a wide amplitude movement, not by passive stretching. The rationale for muscle spindles recalibration is given below.

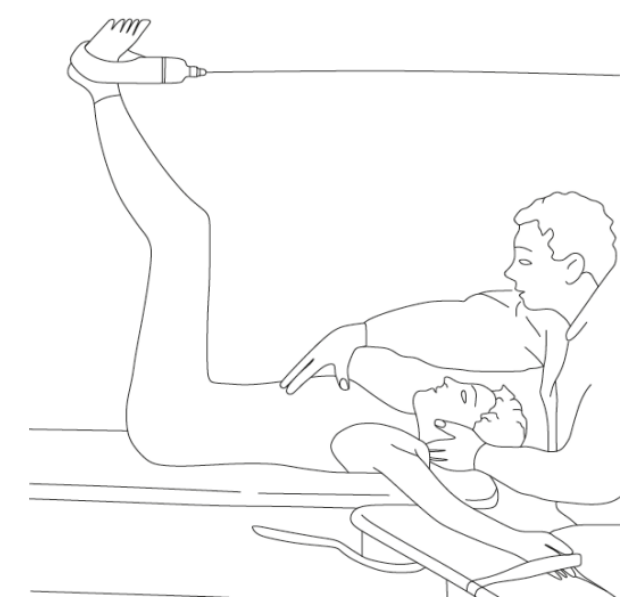

Figure 1. Squared passive position of the body segments for stretching of muscles 
In order to keep the effector - that is body position and movement - detectable by the controller's radar, the population of muscle spindles must signal a background; formalization of the relationship between the controller and the effector is through definition of a reference frame. As Bosco \& Poppele (2001) stated, a primary reference frame for muscle spindles is the spatial domain of the muscle and the coordinate for effective stimuli in that frame of reference is the one-dimensional coordinate of muscle length. From the relationship between muscles and joints, another reference frame for muscle receptors that is joint based can also be determined. Coordinates in this second reference frame would be the relevant joint angles [4], but joint receptors signal their position only at the extremity of joint angles, not at mid-range [5]. This indicates that loading the joint receptors would signal mid-range rotation as well [6].

In order to load the joints, the novel approach expounded here uses elastic tubing to increase the torque of the lower limbs; this coupling is interpreted as impedance - the dynamic relationship between the joint's position and the joint's torque. In a study by Lee et al. (2016), the authors speculate that inappropriate impedance may interfere with the neuron-recovery process. They found that restoring appropriate impedance generates recovery. Lee et al. show however that impedance is regulated during gait, being low during the swing phase and high in the stance phase i.e. when the foot is stationary on the floor [7]. Thus, on the one hand, the simulation of normal impedance is proposed in the stance phase, on the other hand, an effective body position should allow the participant to attempt wide amplitude shifting of the pelvis while loading the lower limbs. For this reason, the kinematic configuration proposed here is a "squared dynamic position", rather than a squared passive position, of the body.

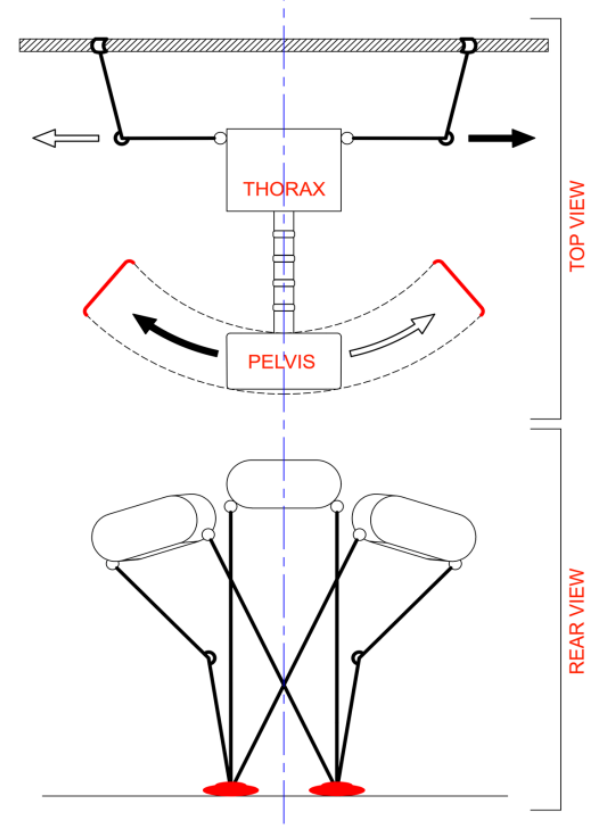

Figure 2. Kinematic configuration of the squared dynamic position of the body. Rear view: thick black line represents the lower limbs and the rounded rectangular object represents the pelvis in its three different positions. Top view: the kinematics of the shoulder girdle; both black and white arrows indicate direction of elbow force contrasting the shifting of pelvis on the contralateral side. Dotted lines in this top view indicate the wide arc of pelvis shifting
The squared dynamic position allows the participant to keep hands firmly on a fixed bar while the feet are stationary on a base of support (see Figure 2). The position then assumed stabilizes the body in a particular way, allowing the participant to attempt wide amplitude shifting of the pelvis. Movements should be slow in order to permit the participant to track the position of the pelvis. Visual feedback is also helpful. Though participants are requested to keep vision horizontal, he or she can also flex the head so as to ascertain the position of the feet and knees. To compare the efficacy of the novel approach in terms of gain in ROM, the standard PNF approach is used. Results obtained in this study put forward a breakthrough approach; the design of equipment used here is open source.

\section{Method and Procedure}

\subsection{Participants}

A total of 56 participants - both genders, BMI normal, mean age 35 (range 21-68 years), exhibiting lumbopelvic extensor stiffness (assessed by performance of the deep squat test) - engaged in two experiments. Many of the younger participants were following a master's degree course in sports science, the remainder were office workers. The inclusion criterion was stiffness in the lumbopelvic extensors, defined as scarce ability to perform the deep squat test. All participants reported being free of cardiovascular conditions and provided written informed consent to participate in the experiment, following a full explanation of procedures pre-approved by the local ethics committee. Much of the experiment was performed in the facilities of the University of Rome Foro Italico, other sessions at John Cabot University, Rome. Twenty-six participants were randomly assigned to Experiment 1 and divided into two groups. One group was treated using the novel approach and the control group with PNF, both for three minutes duration, to compare the improvement in ROM. The remaining thirty participants were assigned to Experiment 2, to evaluate the effect of the novel approach in ameliorating motor disabilities. Data on ROM was collected before and after treatment.

\subsection{Study Interventions}

\section{Experiment 1: Comparison between the novel approach and PNF \\ Experimental treatment (the novel approach)}

Ideally, a quite simple but carefully designed piece of equipment is used to augment impedance of joints. This equipment is in two parts: a plywood base of support with attached elastic tubing plus essentially a cushioned pad, termed the saddle, to which the elastic tubing is coupled. From the squared position, the participant maintains feet stationary on the base of support while the saddle is placed on the level surface of the pelvic-lower lumbar complex. In order to improve control and stability, the participant grips a fixed bar, keeping the hands distant from each other and always in the same place. Total resistance of the tubing extended to one meter corresponds to 734-newton force; this was regulated in ascending fashion. The force 
field generated by the elastic tubing was directed to the base of support - that is, to the participant's feet length and their external distance to each other. A standard distance was chosen, measuring 43 centimetres between the external sides of the feet (see appendix).

From a squared position of the body, using widely spaced hands the participant grips a fixed bar firmly while ensuring that both upper limbs are aligned with the trunk, the lower back adopting a position as close as possible to a table surface. The hips are perpendicular to the ankles in a vertical line. To ensure a comfortable correct position, the simple but specially designed saddle is placed at the pelvis and lower lumbar level, the elastic tubing then being positioned on this saddle. It is advisable to use a timer or a specific number of movements, making this clear for the participant before starting the procedure.

Participants are first shown an illustration of the novel approach, better to understand how to position themselves on the equipment's base of support. The clinician then demonstrates the exercise position and the movement and additional explanations are provided in video (see appendix). The participant then positions him or herself on the equipment. The high level of effort that is required is stressed, as the exercise challenges the body's equilibrium in shifting the pelvis. The participant is also informed that whether he/she wishes to halt they merely need to touch the floor by bending the knees. Further information, which encourages motivation, stresses the short time it takes to perform this approach and its effectiveness in improving ROM on the first attempt - it is very important to continue instruction and feedback to the participant throughout performance of the exercise.

Before starting, the participant must understand the procedure: clarification emphasises that the exercise will challenge the body's equilibrium, resulting in perception of a high level of effort. The clinician must then instruct the participant to grip the bar, keeping both hands firmly in position, better to maintain equilibrium in shifting the pelvis as widely as possible. In this study the procedure lasts three minutes. The clinician assists the participant in performance of the action by placing a hand on segments of the body - e.g.: knees, pelvis and trunk - to help coordination of the dynamics. To provide confidence, the clinician asks the participant first to touch the floor by bending the knees some three times, before asking the participant again to grip the bar and then to perform the pelvis shift, the slow, wide-amplitude, shift of the pelvis. Further assistance can include how to increase stability of the shoulder girdle while performing the pelvis shift.

In order to improve shoulder girdle stability and avoid side-to-side and backward oscillations, the correct position must be assumed, see top view in Figure 2. First, the participant is requested to observe the position of the elbows by turning the head. Then it is explained that, when the pelvis shifts to the side, the elbow opposite must contrast the pelvis movement. From the side, the clinician provides feedback regarding the correct direction of the elbow needed to contrast the pelvis movement. In guiding the participant verbally and physically, the clinician gently pulls the elbow and simultaneously pushes the pelvis. Greater stability is obtained by requesting the participant to keep invariant the distance between the tip of the nose and the bar. A sign/mark on the bar should be used to indicate the midpoint. This procedure helps attain the endpoint of excursion of the pelvis shift, controlled by lateral muscles of the trunk. While these supplementary notes on performance of the approach were not specifically applied in data collection for this manuscript, they are advised for clinical practice.

\section{Control treatment (the PNF approach)}

From a supine position, the participant's limb is raised until it reaches the stiffness barrier, and then held by the clinician for a counteracting force of a few seconds; the limb is then passively stretched towards the chest. Counteracting forces are held for 6 seconds, followed by relaxing the limb extensors and then pulling the limb toward the next barrier for 5 to 10 seconds. Thus, in the first phase, counteracting forces produce an isometric contraction of the limb extensors, while in the second phase the clinician applies a forced stretching of the relaxed limb extensors until the next stiffness barrier is reached. This is performed in three sets, increasing the duration of the second phase up to 30 seconds.

To perform the PNF approach, again an illustration is used in explanation and the approach demonstrated. From the supine position, the leg is raised until the stiffness barrier is reached. The action starts by performing a maximal isometric contraction for 6 seconds, then relaxing the limb so as to apply a forced stretching by pulling it toward the next barrier for 5 to 10 seconds, increasing the duration of the forced stretching up to 30 seconds from the second set.

Experiment 2: lasting effects of the novel approach

In the second experiment, participants responded to an ODI questionnaire prior to, and five to seven days after, attempting the novel approach. To collect data establishing ROM before and after treatment the participant was photographed performing the deep squat test and Kinovea software used, as indicated below.

\subsection{General Procedure}

In both experiments all participants had joint ROM evaluated before and after treatment. To collect data on joint ROM, a deep squat test was used to measure joint angle between leg and thigh. To perform the deep squat test, a standard distance was chosen, measuring 43 centimetres between the external sides of the feet. From the standing position, the participant was required to keep the hands up (towards the ceiling) and elbows locked, he or she then bent the knees while keeping hands up and heels down.

In performing the deep squat test [8], it is essential to keep the hands up and elbows locked in maximal extension, then, as the participant starts to bend the knees, instruction is given to keep the heels down and to bend the knees slowly until the available maximal range of motion is attained. Before performing the test, the participant should familiarize him or herself with the required movement. For this the clinician assists the participant to attempt a deep squat by holding his or her hands, keeping arms parallel to the floor. Afterwards, the participant attempts the maximum deep squat and a photograph is taken for reference. This procedure takes place before, and is repeated after, the treatment.

To collect data, participants were photographed performing the deep squat test and joint ROM calculated 
with Kinovea software [9]. To collect data on motor disability, the ODI questionnaire was used before performing the novel approach and again five to seven days afterwards. A recommended Italian translation version of the ODI questionnaire was used for Italian participants [10], an English ODI validated version for other participants [11]. The ODI questionnaire provides a generic measure for overall health-related quality of life. The questionnaire has been designed to provide information as to how pain can affect a person's ability to manage every-day functional activities. Thus ODI measures chronic-related motor disabilities regarding normal physical activities of daily life.

\section{Data Analysis}

In Experiment 1, data about joint angle was analysed by a $2 \times 2$ factorial ANOVA. Factors were group (two levels, control and experimental) and treatment (two levels, before and after). Post hoc analysis was performed using Tukey HSD test. The overall $\alpha$ level was fixed at 0.05 . To estimate the size of the statistical effects, the partial $\eta$ squared $\left(\eta_{\mathrm{p}}^{2}\right)$ was calculated in addition to probability values. In Experiment 2, paired samples $t$-test was used to verify the efficiency of this novel approach in improve joint angle. Therefore, a $t$-test was performed to understand whether there was a statistically significant amelioration in the participants' motor disabilities score (data obtained with ODI questionnaire) before and after the novel approach. The overall $\alpha$ level was fixed at 0.05 . The overall effect size was calculated with Cohen's $d$. Specifically, Experiment 2 was designed to verify whether such improvements in the degree of ROM could correspond with amelioration in the score of ODI questionnaire.

\subsection{Results of Experiment 1}

Results indicate a significant interaction effect of group per treatment $F_{1,24}=31.83, p<.001, \eta_{\mathrm{p}}^{2}=0.570$. At baseline, the mean joint value was $62.8( \pm 15.2)$ for control, and $62.9( \pm 12.5)$ for experimental group. After treatment, the experimental group mean joint angle value $(42.0 \pm 11.7)$ differs when compared to the control group mean joint angle value $(58.4 \pm 14.2)$. The post hoc test indicates that the treatment's after effect mean value for the RP group was significantly different from all other mean values: RP after (42.0) versus RP before $(62.8)(p<.005)$; RP after versus PNF before (62.9) $(p<.005)$; RP after versus PNF after (58.4) $(p<.05)$, see Figure 3.

\subsection{Results of Experiment 2}

Consistent with Experiment 1, findings indicates statically significant difference in joint angle between before and after treatment, $t(29)=9.48, p<.001, d=1.73$. The mean joint angle at baseline $(72.7 \pm 16.7)$ differs when compared to the mean joint angle after effect treatment (49.9 \pm 19.2$)$, see Figure 4. The amelioration in motor disabilities after treatment was also indicated as significant, $t(29)=7.96, \mathrm{p}<.001, d=1.4$. The mean ODI score at baseline $(8.4 \pm 5.4)$ differs when compared to the mean ODI score after effect treatment $(3.33 \pm 3.8)$, see Figure 5 .

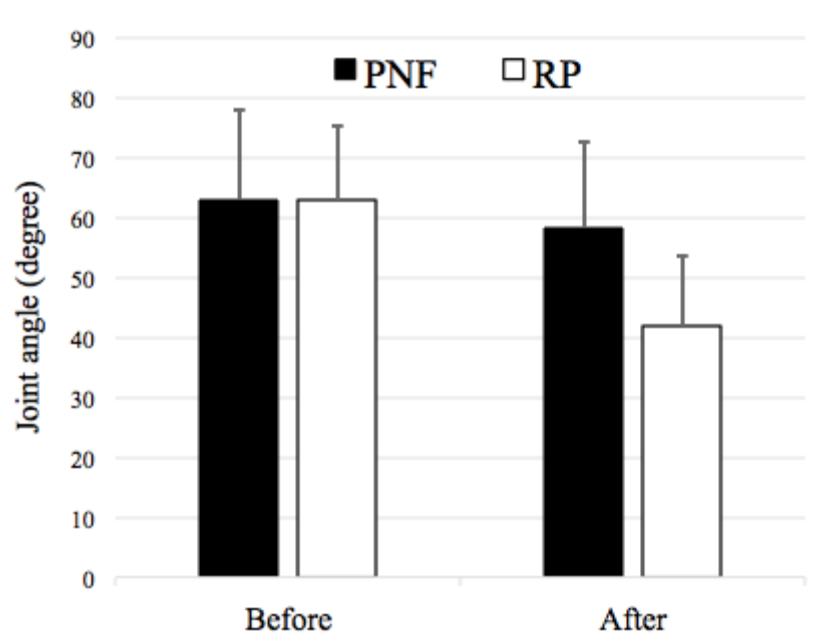

Figure 3. Standard PNF and experimental RP approach comparison, joint angle mean value before and after treatment.

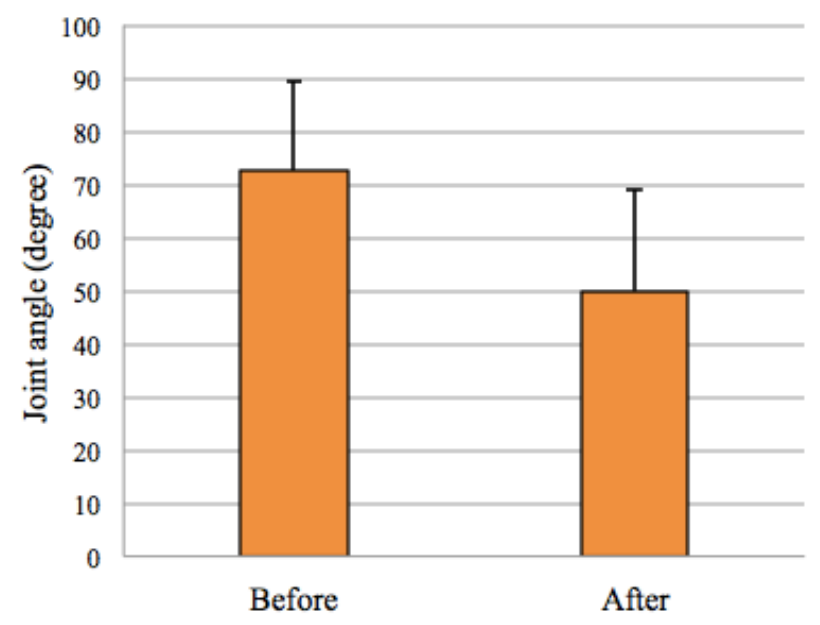

Figure 4. Mean degree of joint angle before and after treatment.

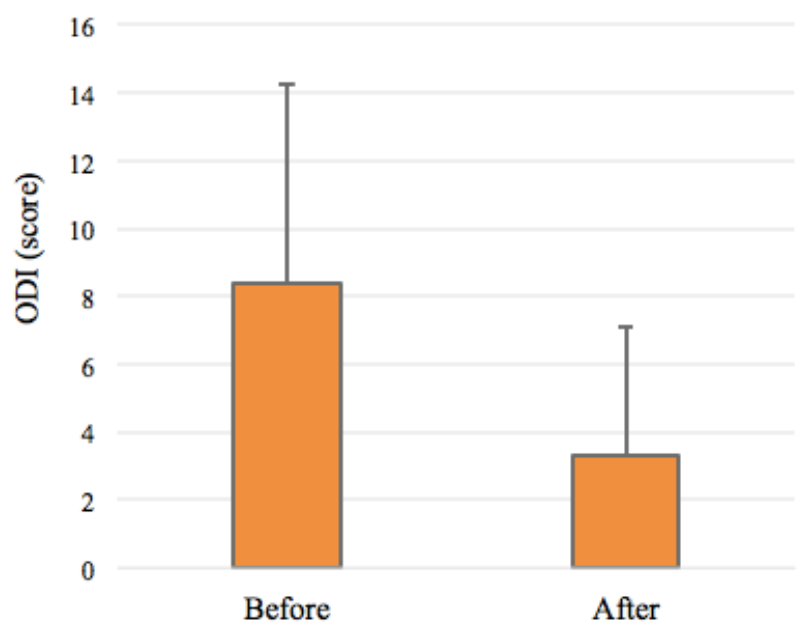

Figure 5. Mean ODI score before and after treatment

\section{Discussion}

Intended for tackling stiffness, passive stretching of muscles is normal practice among both athletes and clinicians. After demonstrating in Experiment 1 the superiority of the novel approach in improving ROM of 
joints when compared to the standard PNF approach, Experiment 2 aimed to verify whether such improvement is associated with amelioration in motor disabilities. A significant amelioration in motor disabilities was indeed confirmed. Moreover, motor disabilities were also evaluated five to seven days after treatment, demonstrating the novel approach's long lasting effect. In addition, the improvement in ROM found in Experiment 1 was repeated in the different group of Experiment 2.

These results raise the question as to whether passive stretching benefits the functioning of the neuromuscular system. Chiba et al. stated that changes in the multisensory system, including the proprioceptive system, elicit immediate changes in posture corresponding to the sensory information, indicating that integration of the various sensory systems are important to control stiffness and body posture [12]. Basically, a muscle's length depends on the activation level of its motoneurones; in turn motoneurones depend on descend commands, but also on the sensitivity of muscle spindles to muscle tension. This shows that stretching of muscles should be undertaken to re-calibrate the muscle spindles, and not merely to stretch muscle tissue. A normal, volitional and instinctive, wide amplitude stretching movement (pandiculation - the classic example being yawning) may well improve ROM of joints, but not the kind of timed, wide amplitude, position adopted in passive stretching. This is because, in timed passive stretching, thixotropy buffers the sensitivity of muscle spindles to muscle tension.

This emphasises the kinematics configuration of the novel approach as compared to normal gait, especially with regard to the dynamics of pelvis shifting. Instead of coordinated rhythmic movement of arms and legs to generate sensory outputs [13], the kinematics here outlined uses a stationary position for hands and feet which facilitates learning of the specific pelvic movement. A determinant factor in design of this novel approach is the knowledge that, during the stance phase of normal gait, one foot is stationary on the ground. In this case, the resultant kinematic is crucial (see Figure 2) because the relation between segments regulates timing of muscle activation and joint torque [14] operating at hip, knee and ankle. These muscles are most active around the start and the end of the stance phase, and virtually inactive during knee extension at mid-stance. Throughout a normal gait, major muscles undergo slow loaded eccentric contractions. Such eccentric contractions represent a powerful input to muscle spindle endings, driving them at high discharge rates especially in contraction against additional resistance. Such eccentric contraction is associated with activation of $\mathrm{\gamma}$ drive, which is important for motor learning [15].

The sensitivity of muscle spindle fibers to muscle tension depends on the body's last passive position and its last dynamics. During functional tasks, actors prefer more comfortable movements performed at intermediate amplitude rather than at wide amplitude [16]. Motor actions are in fact performed at comfortable amplitudes, using many degrees of freedom [17]. This reveals a tendency to avoid the wide amplitude movements which would re-calibrate muscle spindles. In this sense therefore, motor behaviour should be considered a potential intrinsic risk factor, giving rise to the buffering of muscle spindles as a result of thixotropy. In the light of this, certain motor disabilities seem better classified as behavioural motor disabilities. Following this reasoning, such behavioural motor disabilities should be treated by a learning process.

Learning a discrete motor skill, specifically pelvis shifting when the foot is stationery, is important. In a normal gait, one leg accepts the body's weight by shifting the pelvis. This innate ability triggers the sensory consequence responsible for timing a sequence of musculoskeletal reactions. Sensory consequence is the expected proprioceptive feedback, closing the circuitry loop [18]. During performance of the novel approach, the quality and amplitude of the pelvis shifting can easily be verified by comparing sides. That is, different amplitude movements are evidenced comparing sides, assistance being given by the clinician to improve amplitude. By reaching the barrier on the narrow side, the clinician prompts "What if we try to shift the pelvis a little wider to this side? I believe you can do it". Errors and asymmetry must in fact be verbalized clearly in instructions such as "Please check your knee position", "Have you perceived that on this side the movement is smoother?" After attempting wider shifting, participants report a positive sensation of lightness in the lower limbs, describing a positive, effortless, gait. Such a phenomenon is termed sensory attenuation [19], which is the matching between sensory predictions (generated by the controller) and expected sensory consequences (generated by the mechanoreceptors).

Empirically, this novel approach proves efficient even when only light resistance tubing was used to mimicincreased impedance of lower limbs. Rather than related to the tubing-resistance level, the level of effort perceived by participants during execution seems more related to the type of kinematic configuration, especially when performing wider pelvis shifts coordinated with stabilization of the shoulder girdle, as cited above. It would be reasonable to speculate that, when the participant attempts wider pelvis shifting, the unusual dynamics stimulates a population of latent mechanoreceptors causing an equilibrium sensation or a sudden lack of it. This equilibrium sensation, if present, is noticed in the very first attempt at wider shifting, it lessens to almost disappear in successive repetitions as the actor learns the action. During data collection for both the experiments in this study, the resistance level was regulated in ascending fashion, that is, light to moderate in the first two minutes and, after the second minute, the full set of tubing coupled to the saddle. Being a pilot study, more investigation is needed better to understand the relation between the kinematic configuration and the force field generated by performance of the novel approach.

\section{Conclusion}

Based on the evidence, the novel Roman Press proves effective in both improving ROM of joints and ameliorating motor disability. The after effect, sensory attenuation, indicates that the Roman Press is able to ameliorate motor disabilities by re-calibration of the muscle spindles. On a clinical note, since first conception of this novel treatment in November 2016, a still limited, but considerable, number of patient case histories has 
accumulated in clinical practice. Several chronically motor disabled subjects have benefited from use of the Roman Press approach, including for instance patients following radio/chemotherapy: three cancer survivors each reported improved equilibrium and diminished muscle cramps. Many other subjects suffering from a swollen Baker's cyst have shown immediate improvement, including an increased ability to bend the knee. A larger group of subjects complaining of a variety of musculoskeletal disorders from lumbago to neck pain have also reported both immediate and prolonged benefit following treatment.

\section{Conflict of Interest}

The author declares no conflict of interest.

\section{References}

[1] Page P. Current concepts in muscle stretching for exercise and rehabilitation. Int J Sports Phys Ther. 2012 Feb; 7(1): 109-19.

[2] Gregory JE, Wise AK, Wood SA, Prochazka A, Proske U. Muscle history, fusimotor activity and the human stretch reflex. J Physiol. 1998 Dec 15; 513 (Pt 3): 927-34.

[3] Tsay A, Savage G, Allen TJ, Proske U. Limb position sense, proprioceptive drift and muscle thixotropy at the human elbow joint. J Physiol. 2014 Jun 15;5 92(12): 2679-94.

[4] Bosco G, Poppele RE. Proprioception from a spinocerebellar perspective. Physiol Rev. 2001 Apr; 81(2): 539-68.

[5] Burgess PR, Clark FJ. Characteristics of knee joint receptors in the cat. J Physiol. 1969 Aug; 203(2): 317-35.

[6] Grigg P, Greenspan BJ. Response of primate joint afferent neurons to mechanical stimulation of knee joint. J Neurophysiol. 1977 Jan; 40(1): $1-8$.

[7] Lee H, Rouse EJ, Krebs HI. Summary of Human Ankle Mechanical Impedance During Walking. IEEE J. Transl. Eng. Health Med. 2016 4, 1-7.
[8] Cook G, Burton L, Hoogenboom BJ, Voight M. Functional movement screening: the use of fundamental movements as an assessment of function - part 1. Int J Sports Phys Ther. 2014 May; 9(3): 396-409.

[9] Elwardany SH, El-Sayed WH, Ali MF. Reliability of kinovea computer program in measuring cervical range of motion in sagittal plane. Open Access Libr J. 2015 Sep 30; 2(9): 1.

[10] Monticone M, Baiardi P, Ferrari S, Foti C, Mugnai R, Pillastrini P, Vanti C, Zanoli G. Development of the Italian version of the Owestry Disability Index (ODI-I): A cross-cultral adaptation, reliably, and validity study. Spine (Phila Pa 1976) 2009 Sep1; 34(19): 2090-5

[11] van Hooff M, Mannion AF, Staub LP, Ostelo RW, Fairbank JC. Determination of the Owestry Disability Index score equivalent to a "satisfactory symptom state" in patients undergoing surgery for degenerative disorders of the lumbar spine-a Spine Tango registrybased study. 2016 Spine J. 16(10): 1221-1230.

[12] Chiba R, Takakusaki K, Ota J, Yozu A, Haga N. Human upright posture control models based on multisensory inputs; in fast and slow dynamics. Neurosci Res. 2016 Mar 1;104(Supplement C): 96-104.

[13] Nakajima T, Mezzarane RA, Komiyama T, Zehr EP. Reflex control of human locomotion: Existence, features and functions of common interneuronal system induced by multiple sensory inputs in humans. J Phys Fit Sports Med. 2015 May 25; 4(2): 197-211.

[14] Buhrmann T, Di Paolo EA. Spinal circuits can accommodate interaction torques during multijoint limb movements. Front Comput Neurosci. 2014; 8: 144.

[15] Pierrot-Deseilligny E, Burke D. The circuitry of the human spinal cord - spinal and corticospinal mechanisms of movement. 1st edition. Cambridge University Press; 2012, 127-128, 527.

[16] Mark LS, Nemeth K, Gardner D, Dainoff MJ, Paasche J, Duffy M, et al. Postural dynamics and the preferred critical boundary for visually guided reaching. J Exp Psychol Hum Percept Perform. 1997 Oct; 23(5): 1365-79.

[17] Petrovic M, Berg WP, Mark LS, Hughes MR. The impact of object weight, reach distance, discomfort and muscle activation on the location of preferred critical boundary during a seated reaching task. Hum Mov Sci. 2015 Dec; 44: 122-33.

[18] Schimdt RA. A Schema Theory of Discrete Motor Skill Learning. Psychol Rev. 1975 Jul; 82(2): 225-60.

[19] Dewey JA, Knoblich G. Do implicit and explicit measures of the sense of agency measure the same thing? 2014 PLos ONE 9910): e110118. 


\section{Appendix}

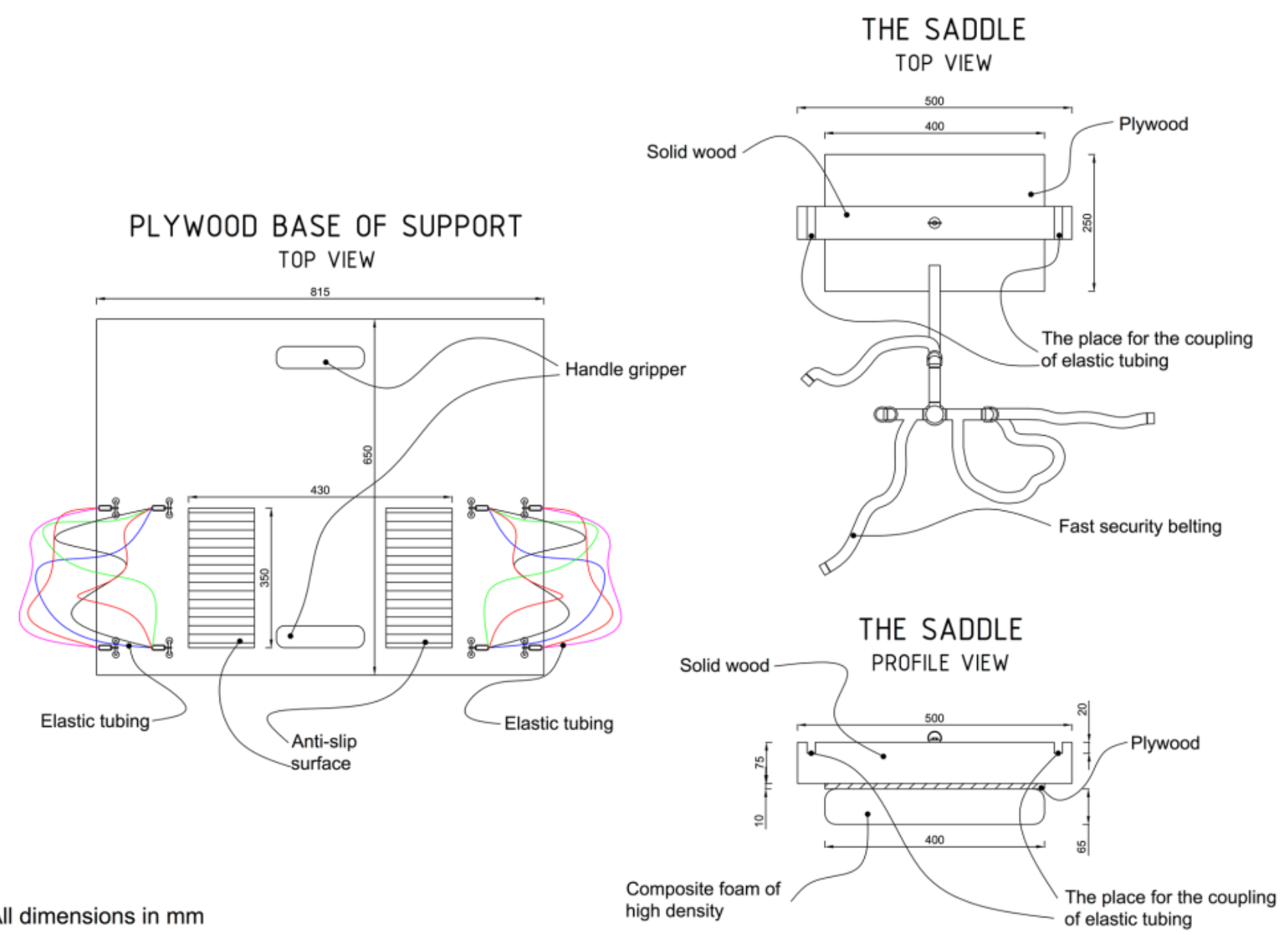

Figure A1. Sketch of the Roman Press 